\title{
Individual decisions in placenta increta and percreta: a case series
}

\section{Frédéric Chantraine*, Michelle Nisolle, Philippe Petit, Jean-Pierre Schaaps and Jean-Michel Foidart}

Department of Obstetrics and Gynecology, University of Liege, Liege, Belgium

\begin{abstract}
Objective: Placenta increta or percreta is an uncommon pathology, sometimes associated with high maternal morbidity. Its prevalence increases proportionally to the number of cesarean sections. This study analyzed the changes of our management strategy to devise treatment guidelines for this uncommon disorder.

Materials and methodology: Between 2005 and 2011, 10 cases of placenta increta or percreta were managed at our university hospital maternity department.

Results: Among the 10 cases, seven were diagnosed prenatally. Two patients were diagnosed early, at 14 and 17 weeks of gestational age, and their pregnancies were terminated. Five had hysterectomies during the intrapartum period, and despite attempted conservative treatment for the two others, hysterectomy proved necessary 2 months postpartum because of intrauterine infections. Seven of the 10 women had hysterectomies.

Conclusion: Prenatal diagnosis of placenta increta or percreta is essential to plan the delivery in a competent tertiary care center. The decision to perform a cesarean hysterectomy or leave the placenta in situ for spontaneous delivery is based on the extent of infiltration, the patient's hemodynamic status, and her desire to remain fertile. The high-risk of infection and severe hemorrhage must not be overlooked should conservative treatment be chosen. This situation requires prolonged close monitoring.
\end{abstract}

Keywords: Embolization; hysterectomy; placenta increta; placenta percreta; postpartum hemorrhage; ultrasound.

*Corresponding author:

Dr. Frédéric Chantraine

Service de Gynécologie-Obstétrique

CHR Citadelle

University of Liège

1, Blv 12ème de Ligne

B-4000 Liège

Belgium

Tel.: +32 42238902

Fax: +3242240361

E-mail: fchantraine@chu.ulg.ac.be

\section{Introduction}

There are varying degrees of abnormal trophoblast infiltration into the implantation site, namely, in increasing order of severity, placenta accreta, placenta increta, and placenta percreta. These implantation abnormalities affect 1 in 1000-2500 deliveries [16, 22].

Placenta percreta is trophoblast invasion of the myometrium to the uterine perimetrium (tunica serosa uteri) and, in certain cases, can exceed this limit and infiltrate into the surrounding organs, e.g., the bladder or bowel. Concurrent with the increasing numbers of scarred uteri, the placenta percreta prevalence has clearly risen over these past few years [6]. It is often associated with placenta previa. Clarke et al. [2] showed that, when placenta previa was present, the risk of placenta accreta increased from $24 \%$ for a patient with one previous cesarean delivery to $67 \%$ for a patient with three or more cesarean deliveries. Specifically, placenta percreta is associated with high maternal morbidity and as much as $7 \%$ maternal mortality [17].

Prenatal diagnosis followed by optimal management at an appropriate tertiary care center is essential to limit the devastating maternal consequences and to decide the most appropriate surgical strategy: conservative management with the placenta left in situ after cesarean delivery, or hysterectomy, possibly associated with extensive pelvic surgery. The former therapeutic approach exposes the woman to prolonged risks of hemorrhage and endometrial infection or sepsis, whereas the latter may prove extremely difficult to achieve when percreta placentation is associated with parametrial invasion.

The purpose of this study was to retrospectively analyze the last 10 patients diagnosed with placenta increta or percreta and treated in our university hospital maternity department and to evaluate their outcomes in comparison to those in the literature. Based on our own experience and a literature review, several clinical recommendations are proposed.

\section{Patients and methods}

Our tertiary care maternity unit performs 2300 deliveries annually and is the referral center for high-risk pregnancies from a network of maternity hospitals performing 15,000 deliveries a year.

This retrospective study included all 10 cases of placenta increta and percreta treated between June 2005 and June 2011. Clinical information was obtained from the written delivery reports and databases of the Pathology and Radiology Departments. Data from intrahospital management and examinations performed in referring centers were compared and analyzed on a case-by-case basis.

Placenta increta and percreta were suspected based on ultrasonography, followed by magnetic resonance imaging (MRI), verified 
during cesarean delivery, and confirmed by histopathological examination of surgical hysterectomy specimens.

\section{Results}

The demographic and clinical characteristics of the 10 women are reported in Table 1. All of them presented risk factors for abnormal placental implantation: previous cesarean delivery, curettage or other uterine surgery, or placenta accreta. Notably, for seven women, six of whom had with more than one cesarean delivery, placenta percreta was diagnosed prenatally. Placental insertion was anterior and previa in seven of 10 cases. Patient 6's placenta invaded the fundus of the uterus, probably linked to her postpartum curettage after a previous vaginal birth. The infiltration of the surrounding tissues and/or vesicouterine fold was confirmed intraoperatively for patients 1, 2, 4, and 5. Their placentas were left in situ, after their fetuses had been extracted through a vertical fundal hysterotomy. Patient 10 had placenta percreta infiltrating the posterior and fundal walls of the uterus, which had been suspected preoperatively and was confirmed during laparotomy, when infiltration of the mesocolon was also diagnosed. After fetal delivery, the uterus with the placenta in situ and $30 \mathrm{~cm}$ of the sigmoid colon were resected en bloc (Figure 1).

In total, seven hysterectomies were performed: four during cesarean deliveries (two because of massive intraoperative bleeding and two were elective), one several hours after massive hemorrhaging, and two were done 2 months after cesarean deliveries because of intrauterine infection and pelvic abscess, respectively.

Patients $2-5,8$, and 9 received blood transfusions (mean, 7 units; range, 2-13 units). Patients 3, 8, and 9 experienced massive hemorrhage when manual placental removal was attempted. The details of therapeutic management are given in Table 2, with cases listed in chronological order.
Table 1 Characteristics of the 10 women with placenta percreta.

\begin{tabular}{lc}
\hline Characteristic & Value $^{\mathrm{a}}$ \\
\hline Age & $30.0(24-37)$ \\
Gravidity & $4.2(2-7)$ \\
Parity & $2.4(1-5)$ \\
Risk factors & $10 / 10$ \\
$\quad$ Previous manual placental delivery & $2 / 10$ \\
Previous curettage & $4 / 10$ \\
At least one previous cesarean & $7 / 10$ \\
Prenatal diagnosis & $7 / 10$ \\
Complementary MRI & $7 / 10$ \\
Parametrial, bladder, or bowel invasion & $6 / 10$ \\
\hline
\end{tabular}

${ }^{a}$ Values are expressed as mean (range) or number.

\section{Discussion}

The real prevalence of placenta accreta cannot be determined with certainty. Indeed, simple placenta accreta is most often resolved by manual delivery for incomplete separation. No histopathological confirmation can be made in these situations, for which obstetrical management is standard and well established. In their report on 310 cases of placenta accreta, Gielchinsky et al. [4] also cited the difficulty of making this diagnosis. Therefore, we analyzed only our case series with placenta increta or percreta because the clinical signs and prenatal diagnosis are more obvious when the ultrasonographer pays particular attention to the implantation site. Placenta increta or percreta is less common than placenta accreta but is responsible for greater morbidity and even maternal and fetal mortality [17].

The most troubling ultrasound signs of placenta percreta are the absence of retroplacental myometrium, intraplacental basal hypoechogenic lacunae, and high-flow, low-resistance vascularization (color and pulsed Doppler) [18]. All these
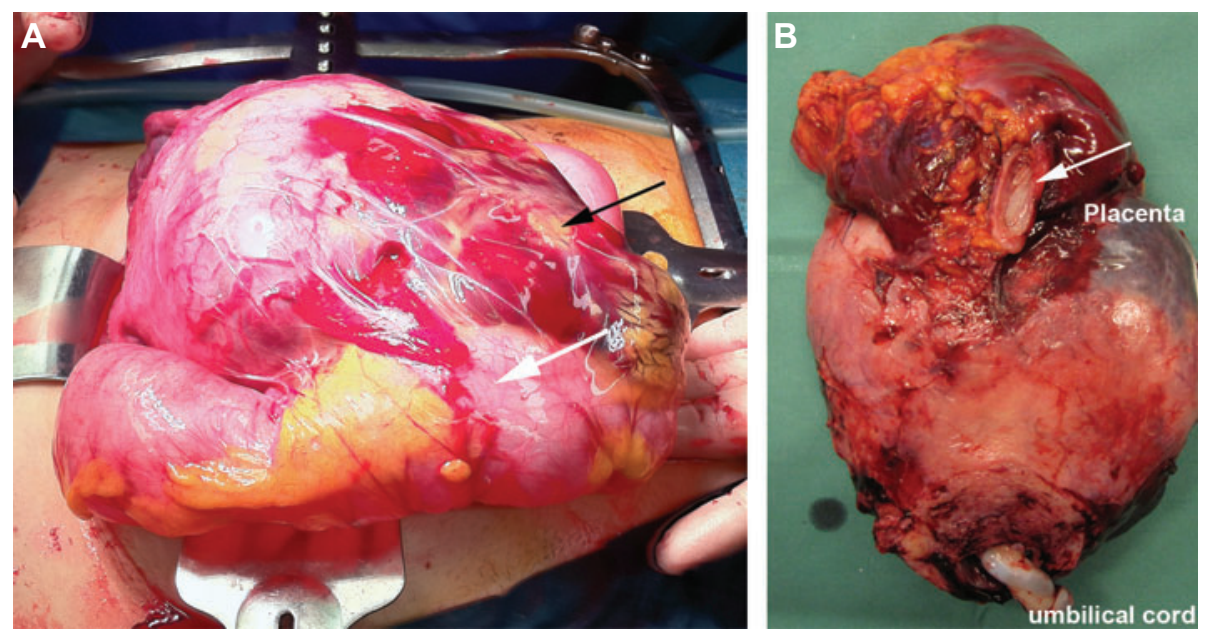

Figure 1 Case 10.

(A) Posterior and fundal placenta percreta invading the uterine serosa and adherent to the sigmoid colon (white arrow). This photo was taken after fetal delivery. The black arrow indicates placenta percreta infiltration of the mesocolon. (B) Surgical specimen showing the en bloc resection of the uterus and $30 \mathrm{~cm}$ of sigmoid colon. The white arrow points to one end of the sigmoid colon. 


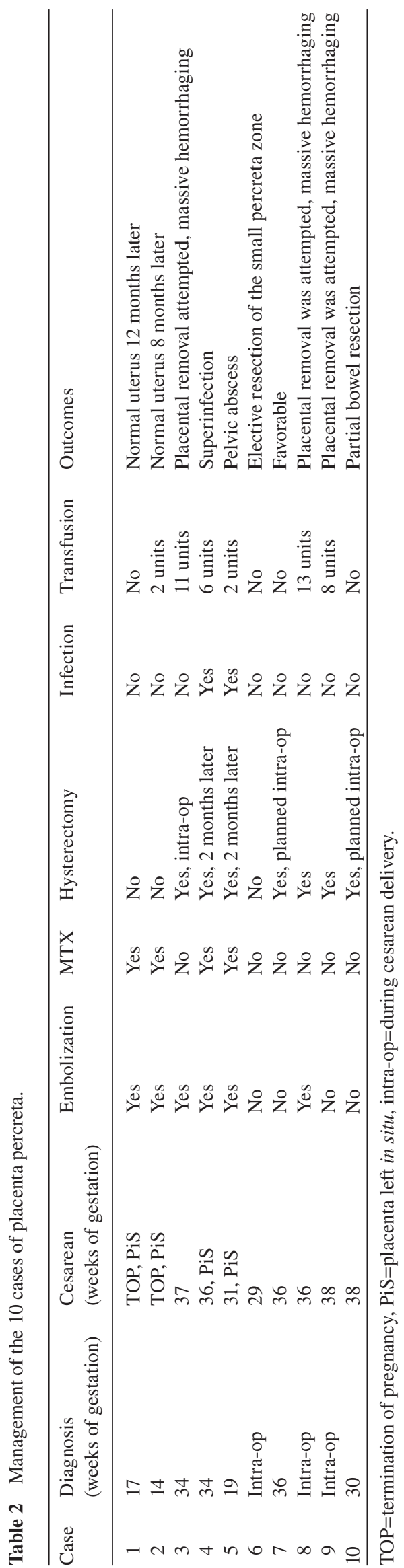

signs were observed in the seven patients with prenatal diagnoses (Figures 2 and 3). In the context of previous cesarean delivery and previa, anterior placentation, those three ultrasound signs are actively sought, which might account for the high percentage of prenatal diagnoses [13].

For patients diagnosed during prenatal ultrasonography, complementary MRI (Figure 2) was carried out to evaluate the lateral extension and depth penetration of the placenta. In our opinion, correspondence between the findings of the two imaging techniques is essential to choose between the two possible therapeutic approaches: cesarean delivery combined with hysterectomy or cesarean delivery with the placenta left in situ. This approach facilitates planning the delivery, especially in terms of selecting the sites for the cutaneous and uterine incisions.

The diagnoses of placenta percreta in patients 1 and 2 were suspected early, at 17 and 14 weeks of gestation, respectively, based on ultrasound findings and MRI. After methotrexate (MTX) injection (Teva Pharma, Wilrijk, Belgium) and bilateral uterine artery embolization, their fetuses were extracted through fundal hysterotomy at 20 and 18 weeks of gestation, respectively. Their placenta percreta diagnoses were definitively confirmed during surgery, and each patient's placenta was left in situ. In addition to placenta percreta, patient 1 also had anhydramnios due to the premature rupture of the amniotic sac. Patient 2's request for termination of pregnancy was accepted by the Ethics Committee, in light of the extensive placental infiltration, which was likely to deteriorate further with advancing pregnancy. Notably, several cases of uterine rupture and massive hemorrhaging have been reported for placenta percreta diagnosed at the beginning of the second trimester $[3,12,14]$.

It could be hypothesized that placenta increta or percreta is the continuation of untreated cesarean scar pregnancy (CSP), but it must be kept in mind that different CSP types exist $[1,23]$. First, real CSP, with deep implantation into the scar defect, is at high-risk of spontaneous uterine rupture. Second, when the implantation is "on" or close to a previous cesarean section scar, the pregnancy may progress to a viable birth by taking the risk of life-threatening hemorrhage due to placenta accreta, increta, or percreta. In our opinion, placenta percreta in patients 1 and 2 could be attributed to that latter CSP type. Because our management of those cases started at 16 and 17 weeks (second trimester), we prefer the term "placenta percreta," rather than CSP.

Four patients underwent cystoscopy, during which absence of infiltration of the bladder mucosa could be ascertained. Thus, this semi-invasive examination failed to provide additional information. In their review of 54 cases of placenta percreta with bladder infiltration diagnosed with ultrasound and MRI, Washecka and Behling [25] reported the failure of cystoscopy to prenatally detect bladder mucosa infiltration. In response to those observations, our department no longer orders cystoscopy for the diagnostic workup of a patient with deep placental invasion into the anterior uterine wall and bladder except when macroscopic hematuria is present.

Our clinical approach involves planning deliveries of placenta percreta at $\sim 36$ weeks' gestational age to prevent maternal morbidity due to the spontaneous onset of labor 


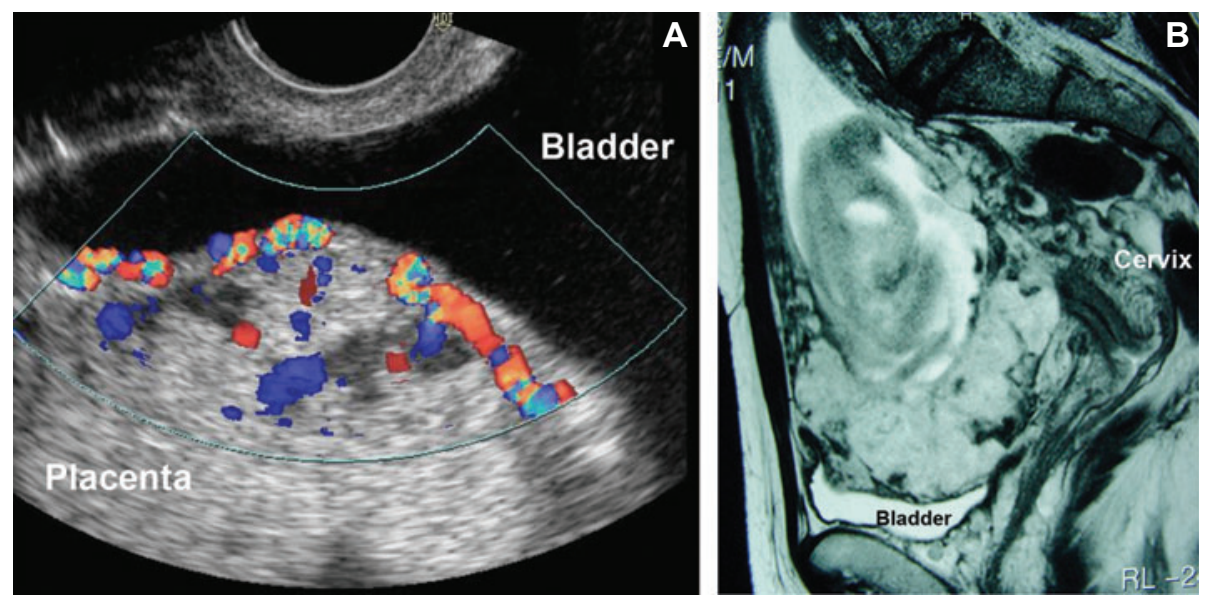

Figure 2 Case 2.

(A) Transvaginal ultrasound showing the anterior and previa placenta percreta exhibiting the following ultrasonographic signs of abnormal placenta infiltration: thinning of the myometrium (virtually absent here), placenta lacunae, and turbulent blood flow. (B) MRI (T2-weighted sagittal view) showing placenta percreta and previa implanted in the lower anterior wall of the uterus (fetus in situ).

and delivery. An unscheduled emergency delivery in such cases would also markedly increase neonatal risks and morbidity. This strategy is supported by the findings of Warshak et al. [24], who showed less maternal blood loss but increased risk of prematurity, admission to neonatology units, and prolonged hospital stays when delivery was planned at 34-35 weeks' gestation. Furthermore, O'Brien et al. [17] showed that, beyond 36 weeks of gestation, the risk of spontaneous bleeding and emergency delivery increased. The optimal time of delivery depends on several factors, e.g., placental location, cervical length, bleeding episodes, history of preterm delivery. Individual planning and management is recommended to determine the optimal week of delivery for every patient.

In our series, after fetal extraction, the placenta was left in situ in four women. This strategy was chosen because of the diffuse infiltration observed, with parametrial involvement and

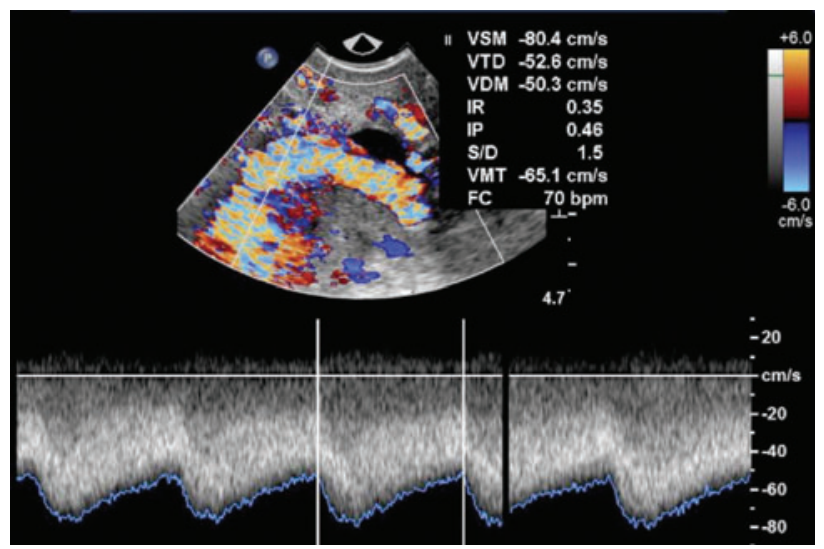

Figure 3 Case 5.

Transvaginal ultrasound showing anterior low-lying placenta percreta. The pulsed Doppler shows the high-volume, low-resistance blood flow. invasion of the bladder wall. Cesarean hysterectomy would have been difficult technically in the absence of a dissection plane and carried a high-risk for the woman. These patients also wanted to retain fertility. Several publications described the possibility of deferred spontaneous vaginal delivery or resorption of the placenta left in situ [7, 11, 17]. However, for our four patients, no spontaneous placental delivery occurred. Total resorption was associated with mild prolonged metrorrhagia, lasting 12 and 18 months, respectively, for patients 1 and 2. Patients 4 and 5 developed infections following cesarean delivery, necessitating hysterectomies 2 months later, which were subjectively more easily performed than at the time of delivery (Figure 4). This risk of infection associated with the placenta in situ is well documented in the literature $[5,10]$.

Patients 7 and 10 underwent planned cesarean hysterectomy because the placenta had not penetrated beyond the perimetrium (case 7) and cleavage planes for a hysterectomy were maintained (both cases). These patients no longer wanted to remain fertile and requested one-step surgery, if possible.

Forced manual removal of a deeply infiltrating placenta must be avoided to prevent uncontrolled massive hemorrhaging, as what occurred in patients 3,8 , and 9. All three required multiple blood transfusions (8-13 units) and, finally, hysterectomy.

The uterine arteries of patients $1,2,4$, and 5 were prophylactically embolized just after cesarean delivery to prevent major and unpredictable postpartum bleeding (Figure 4). Such systematic preventive embolization without bleeding has to be considered carefully. Indeed, for patients 2, 4, and 5, it did not prevent major hemorrhaging or the need for second embolizations. This failure might be the consequence of the extensive lacunae in the myometrium [19] with high blood flow, rendering 500- $\mu \mathrm{m}$ polyvinyl alcohol particles useless. Such prophylaxis is no longer performed systematically in our department. However, therapeutic embolization remains 


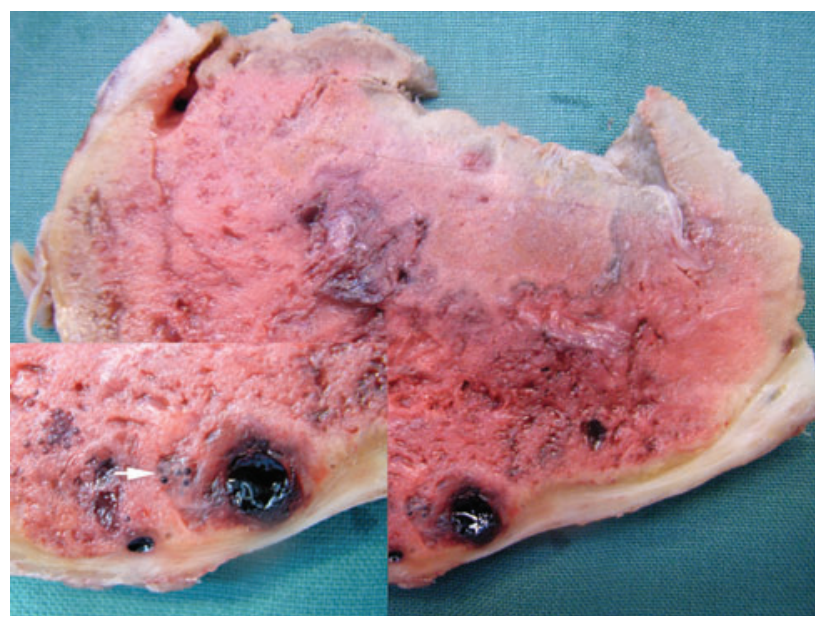

Figure 4 Case 4.

Hysterectomy specimen obtained 2 months after fetal delivery with placenta left in situ (indication for hysterectomy: endometrial and pelvic infection). Inset: cross-section shows the deep placental infiltration and the persistent presence of uterine artery embolization particles (white arrowhead), administered immediately after delivery, that were unable to adequately fill the enlarged vascular lacunae and stop the hemorrhaging.

indicated to manage severe hemorrhaging in other obstetrical circumstances, e.g., uterine atony or birth canal lacerations.

MTX was prescribed as adjuvant therapy to accelerate placental involution for the four patients whose placentas were left in situ. The doses to be administered are not standardized $[7,9,18]$, and no studies have yet elucidated the risks or benefits of this treatment [18]. Dramatic complications, including maternal death, were reported after injecting MTX into the umbilical cord [20]. Among our four patients treated with MTX, two developed infectious complications, which might be attributable to the immunosuppressive action of MTX. In light of these results, our patients with placenta percreta left in situ are no longer given MTX. Progressive regression of active trophoblast is monitored by measuring plasma human chorionic gonadotropin levels at weekly intervals, biological markers of inflammation, and physical examination.

The choice between radical cesarean hysterectomy or the more conservative placenta in situ therapeutic strategy for placenta percreta must be made based on the extent of invasion of the parametrium or adjacent organs (bladder and/or bowel). If placental trophoblast does not penetrate beyond the perimetrium, which sometimes can only be definitively determined intraoperatively, cesarean hysterectomy without placental removal seems to be the best solution offering the least maternal morbidity. If, however, the placenta has infiltrated the perimetrium and surrounding tissues, avoiding placental extraction is recommended. The risk of massive hemorrhaging during forced delivery or hysterectomy without classical anatomical reference points is very high. Severe placenta percreta with invasion of adjacent organs or parametrium remains a very dangerous situation for patients, given its associated non-negligible morbidity and even mortality $[17,20]$. In such instances, delayed hysterectomy can be performed 4-6 weeks after cesarean delivery. This lag time allows the periuterine vascularization to recede and prevents late onset massive hemorrhaging or severe infection.

Prenatal diagnosis is of course imperative. Based on our experience and the literature, it should be possible, especially for placenta increta or percreta $[8,15,21]$. The placental implantation site must be explored carefully, especially when the placental insertion is anterior and previa and the patient has undergone a previous cesarean delivery. If an implantation abnormality is suspected, the patient must be referred to an experienced team to confirm the diagnosis. Delivery must then be planned in an institution that can manage the serious complications of this pathology. Postpartum hemorrhage may occur early or late and can be unexpected, sudden, and severe. Infection of the placenta left in situ, with its possible progression to infectious toxic shock and hemorrhage, is another major issue to be considered and that requires close monitoring.

During the 5 years that these placenta increta and percreta patients were managed in our department, we instigated the following five changes. Active systemic screening for implantation anomalies during routine prenatal ultrasonography has become standard practice and is done thoroughly. When possible, elective cesarean hysterectomy at 36-37 weeks of gestational age is preferred to prevent the severe complications associated with postpartum placenta in situ. The uterine arteries are embolized only when postoperative bleeding occurs. MTX is no longer given systematically, as it appears to facilitate infectious complications. A multidisciplinary team has been established to ensure the management of placenta increta and percreta according to standardized guidelines.

\section{Conclusion}

The frequency of placenta increta and percreta is increasing in direct relationship to the higher number of cesarean deliveries and other types of uterine surgery. Prenatal diagnosis is possible but requires thorough examination by an experienced ultrasonographer, who actively looks for this pathology in a patient with an anterior low-lying or previa placenta and a history of cesarean delivery or postpartum curettage. This prenatal detection should provide accurate information. The patient can then be fully informed of her therapeutic options and their potential consequences and be optimally prepared for delivery by an experienced team. Prenatal diagnosis does not necessarily avoid hysterectomy but can ensure reduction of maternal risks.

A decision between radical and conservative strategies for placenta increta and percreta must be made based on the degree of placental infiltration (whether it goes beyond the perimetrium and into adjacent organs) and other variables: the patient's hemodynamic status and her desire to remain fertile. In our opinion, cesarean hysterectomy when the perimetrium is intact remains the best therapeutic option to treat placenta increta and percreta. If, however, placental invasion largely exceeds the perimetrium and/or the patient wishes to remain fertile, management of the placenta in situ is indicated, with 
full knowledge of the risks of major hemorrhaging and severe infection.

The benefits of prophylactic embolization of the uterine arteries and adjuvant MTX administration for placenta in situ are not clear and remain controversial.

Multidisciplinary management (obstetrics, surgery, interventional radiology, anesthesia, etc.) in a referral center is indispensable for a favorable outcome.

\section{References}

[1] Ash A, Smith A, Maxwell D. Caesarean scar pregnancy. Br J Obstet Gynaecol. 2007;114:253-63.

[2] Clark SL, Koonings PP, Phelan JP. Placenta previa/accreta and prior cesarean section. Obstet Gynecol. 1985;66:89-92.

[3] Esmans A, Gerris J, Corthout E, Verdonk P, Declercq S. Placenta percreta causing rupture of an unscarred uterus at the end of the first trimester of pregnancy: case report. Hum Reprod. 2004;19:2401-3.

[4] Gielchinsky Y, Rojansky N, Fasouliotis SJ, Ezra Y. Placenta accreta - summary of 10 years: a survey of 310 cases. Placenta. 2002;23:210-4.

[5] Guillot E, Raynal P, Fuchs F, Lepercq J. Échec d'un traitement conservateur d'un placenta accreta. Gynecol Obstet Fertil. 2006;34:1055-7.

[6] Hamilton BE, Martin JA, Ventura SJ. Births: preliminary data for 2005. Natl Vital Stat Rep. 2006;55:1-18.

[7] Henrich W, Fuchs I, Ehrenstein T, Kjos S, Schmider A, Dudenhausen JW. Antenatal diagnosis of placenta percreta with planned in situ retention and methotrexate therapy in a woman infected with HIV. Ultrasound Obstet Gynecol. 2002;20: 90-3.

[8] Japaraj RP, Mimin TS, Mukudan K. Antenatal diagnosis of placenta previa accreta in patients with previous cesarean scar. J Obstet Gynaecol Res. 2007;33:431-7.

[9] Kayem G, Davy C, Goffinet F, Thomas C, Clement D, Cabrol D. Conservative versus extirpative management in cases of placenta accreta. Obstet Gynecol. 2004;104:531-6.

[10] Kayem G, Anselem O, Schmitz T, Goffinet F, Davy C, Mignon A, et al. Comparaison historique de deux types de prises en charge de placenta accreta: radicale versus conservatrice. J Gynecol Obstet Biol Reprod. 2007;36:680-7.

[11] Legro RS, Price FV, Hill LM, Caritis SN. Nonsurgical management of placenta percreta: a case report. Obstet Gynecol. 1994;83:847-9.

[12] LeMaire WJ, Louisy C, Dalessandri K, Muschenheim F. Placenta percreta with spontaneous rupture of an unscarred uterus in the second trimester. Obstet Gynecol. 2001;98:927-9.
[13] Levine D, Hulka CA, Ludmir J, Li W, Edelman RR. Placenta accreta: evaluation with color Doppler US, power Doppler US, and MR imaging. Radiology. 1997;205:773-6.

[14] Medel JM, Mateo SC, Conde CR, Cabistany Esque AC, Rios Mitchell MJ. Spontaneous uterine rupture caused by placenta percreta at 18 weeks' gestation after in vitro fertilization. J Obstet Gynaecol Res. 2010;36:170-3.

[15] Megier P, Gorin V, Desroches A. Placentas bas insérés échographiquement au $3^{\mathrm{e}}$ trimestre de la grossesse: recherche de signes de placenta accreta/percreta et de vaisseaux praevia. J Gynecol Obstet Biol Reprod. 1999;28:239-44.

[16] Miller DA, Chollet JA, Goodwin TM. Clinical risk factors for placenta previa-placenta accreta. Am J Obstet Gynecol. 1997;177:210-4.

[17] O'Brien JM, Barton JR, Donaldson ES. The management of placenta percreta: conservative and operative strategies. Am J Obstet Gynecol. 1996;175:1632-8.

[18] Oyelese Y, Smulian JC. Placenta previa, placenta accreta, and vasa previa. Obstet Gynecol. 2006;107:927-41.

[19] Schaaps JP, Tsatsaris V, Goffin F, Brichant JF, Delbecque K, Tebache M, et al. Shunting the intervillous space: new concepts in human uteroplacental vascularization. Am J Obstet Gynecol. 2005;192:323-32.

[20] Sentilhes L, Ambroselli C, Kayem G, Provansal M, Fernandez $\mathrm{H}$, Perrotin F, et al. Maternal outcome after conservative treatment of placenta accreta. Obstet Gynecol. 2010;115:526-34.

[21] Shih JC, Palacios Jaraquemada JM, Su YN, Shyu MK, Lin CH, Lin SY, et al. Role of three-dimensional power Doppler in the antenatal diagnosis of placenta accreta: comparison with grayscale and color Doppler techniques. Ultrasound Obstet Gynecol. 2009;33:193-203.

[22] Timmermans S, van Hof AC, Duvekot JJ. Conservative management of abnormally invasive placentation. Obstet Gynecol Surv. 2007;62:529-39.

[23] Vial Y, Petignat P, Ohlfeld P. Pregnancy in a cesarean scar. Ultrasound Obstet Gynecol. 2000;16:592-3.

[24] Warshak CR, Ramos GA, Eskander R, Benirschke K, Saenz CC, Kelly TF, et al. Effect of predelivery diagnosis in 99 consecutive cases of placenta accreta. Obstet Gynecol. 2010;115:65-9.

[25] Washecka R, Behling A. Urologic complications of placenta percreta invading the urinary bladder: a case report and review of the literature. Hawaii Med J. 2002;61:66-9.

The authors stated that there are no conflicts of interest regarding the publication of this article.

Received June 23, 2011. Revised December 17, 2011. Accepted January 3, 2012. Previously published online January 23, 2012. 